\title{
Detection of Brain Tumor in EEG Signals Using Independent Component Analysis
}

\author{
Akram Rashid*1, Seema Tahir, ${ }^{2}$ Aamer Saleem Ch. ${ }^{3}$ I.M. Qureshi ${ }^{4}$
}

\author{
Accepted 15 ${ }^{\text {th }}$ August 2014
}

\begin{abstract}
The Electroencephalogram(EEG) is Scientifically becoming an important tool of measuring brain activity. The EEG data is used to diagnose brain diseases and brain abnormalities. EEG helps to suit the increasing demand of brain tumor detection on affordable prices with better clinical and healthcare services. This research work presents a technique of efficient brain tumor detection in EEG signals using Independent Component Analysis(ICA). EEG signals which actually are carrying information regarding brain abnormalities are also contaminated by the artefacts both from subjects and equipment interferences. Artefacts are removed using adaptive filtering techniques(ICA). The signal features are extracted by ICA which are buried in wide noise band. This clean artefact free EEG signal is then used as a train input for Maximum Likelihood Detector. The trained input is then fed with test EEG signals. This way the presence of brain tumor in EEG signal is effectively detected. The results obtained experimentally demonstrate the efficiency of the technique in removing artefacts from EEG signals for efficient of brain tumor detection.
\end{abstract}

\section{Keywords: Elimination, Electroencephalogram, Image processing, Diagnose, Brain, abnormalities, Detection, Signals Detector.}

\section{Introduction}

The information regarding the cerebral cortex nerve cells of brain produce electrical activity. Electroencephalogram (EEG) collect these electrical signal information via electrodes. The clinical assessment of brain activities is being carried out by analyzing recorded EEG signals. The ocular artifacts have been corrected by applying the methods but the study shows that even after application of correction methods there still remains noisy activity in the form of eye blink artifact in real EEG data. After the maximum blink excursion the occipital topography peaks at about $255 \mathrm{~ms}$. This is the replica of the Berg's findings [9]. Since the residual remains after the application of either correction so it cannot be explained just by any overcorrection method. Moreover simulated data also does not give any information about that. Also different results are obtained with respect to the different procedures applied and the position of the electrodes on the scalp of the patients. This gives different mutual information of the residual activity, EEG and vertical EOG which occur at the maximum eye blink. These effects are shown in simulated data.

\subsection{Real Data}

The regression approach which has been taken without average subtraction of raw EMCP w/s and extended infomax with respect to the mean mutual information gives quite similar results. It is notable that the EMCP w/s and extended infomax are significantly different from each other. The difference is very significant statistically. It is in terms of marginal absolute values. Always It is considered the result of the different eye blinks of the patient. For ICA a source is required to show frequent activation because ICA

\footnotetext{
${ }^{1,2,4}$ Faculty Department of Electrical Engineering Air University Islamabad, Pakistan akram@mail.au.edu.pk

${ }^{3}$ Faculty EE Department University of Lahore, Islamabad Campus Note: This paper has been presented at the International Conference on Advanced Technology \& Sciences (ICAT'14) held in Antalya (Turkey), August 12-15, 2014.
}

is a statistically based procedure. It is required for extraction of the required components reliably.

We can define the EMCP w/s alternatively that overcorrection of frontal and occipital channels lead by EMCP w/s. Alternately we can sat that the pure blink activity is filtered by extended infomax. There remains other artifacts such as arise from vertical eye movements or muscle and reopening of the eye of the patient. Since only blinks are unambiguously identifiable this components Identification becomes difficult. These are which are responsible for ocular artifacts. The mean and the mutual information about residual was higher with raw average subtraction EMCPs it was for the regression technique. It is observed that it was the highest for uncorrected data. A large residual activity is the EMCPs results after application for which are actually the averages of eye blink and also the maps of topographic previously studied blink maximum. It is important to note ICA generate two components for blink activity. The above analysis shows that under some circumstances the ICS may give results which are actually lying in a higher dimensional decomposition as are usually predicted for the EEG components. Because of this characteristics a detailed investigations is required. Whatever the correction procedure we are using after eye blink maximum. At about $260 \mathrm{~ms}$ we analyzed in the time window a large positivity. This shows the replication of the Berg's findings [10]. His findings was that residual results when the eye was reopen. This way induces a strong charge of input for visual and as a result visual evoked ERP is produced. So the residual has strong physiological origins.

We can conclude that if the corrections have been made in EEG by any correction algorithm then residual must be taken into account. This will be our future research target whether residual can be removed using ICA or in what extent residual is going to contributes in estimated ERP. In different correlation procedures residual activity varied differentially. We obtain same results from both Component and Propagation models for ICA. These models provides the clean uncontaminated data as in simulation. The data 
which was corrected is highly correlated with uncontaminated clean simulated data. The EMCP w/s clearly follow the findings. But it is not strictly followed by EMCPs. The EMCPs, EMCP w/s and ICA always follow the correlation residual activity.

The EMCPs amplitude failed if the patient is constantly blinking eyes. This is because the average blink, like uncorrelated, showed the similar activity in time-course. When the blink amplitude varied then corrections works better for component model. These analysis are both for components model and propagation model as well. In component model if the amplitude of eye blink did not changing fast then the EMCP w/s gives comparatively better results for ICA. Results are inferior to ICA when eye blink amplitudes are varying blink amplitude. The Conclusion is that for blink maximum there are not better results whether we are following any method. In this case this shows that potential we analysed is physiological affecting results eyes are opened and reopened time and again.

When there is no variations in blinks duration and in amplitude then at blink time point the vertical EOG gives a blink signal a good estimate. When the duration and the amplitude are varying then the estimations will be imprecise approximations and will be taken by regression analysis. This is why in the real data ICA and $\mathrm{EMCP}$ w/s give analogous results. The regression error is small in spontaneous blinks because here the duration as well as the amplitude does not vary strongly. Here comes the question which simulation is good for an appropriate model. In present research data shows that component model works better, because for simulated and real data that we obtain the analogous correction procedure results.

We can conclude that findings in real data are strongly supported by simulations. The ICA technique was equivalently effecting both in component as well as propagation model. In both the cases corrected data by ICA was resembling in both models with the uncontaminated time course clean data. While in EMCP-corrected data results were not matching. In both models there was a large residual activity. This means blinks constructed by a propagation model and realized by a component model were carrying large residual activity and residual were varying with the eye blink amplitude variations as shown in simulations.

It is important to note that simulation results are corresponding with mutual information. So for evaluation criterion the mutual information can be used effectively. The basic advantage is that the distribution parameters electrical activity of EEG and EOG are independent and hence the technique is best suited for ICA implementation.

\subsection{Data Recorded by EEG}

A 57 channel system is used to record EEG data. A Quick Amp 72

is used for relative to average reference. The data is of the following Channels (AF4, AF8, FPz, FP1, AFz, AF7, AF3, , FCz, FT9, FC5, FC3, FC1, FC2, FC4, FP2, Fz, F7, F3, F4, T7, C5, C3, C1, F8 FC6, FT1, C2, C4, C6, T8, CPz, CP5, CP3, Pz, P7, P3, P1, P2, P4, P8, Oz, PO9, PO7, PO3, PO4, CP1, CP2, CP4, CP6, PO8, $\mathrm{PO} 1$, , I1, I2, Cz Oz, O1, O2) were positioned following the 10-20system (Jasper, 1958).

We had placed two additional electrodes M2,M1 and four electrodes were recording EOG signals $\mathrm{SO} 2$ was above right eye $\mathrm{IO} 2$ positioned below LO1 \& LO2 were at outer acanthi. Average reference was used for all channel recordings. Frequency $500 \mathrm{~Hz}$ was sampling rate and impedance was less than $10 \mathrm{k} \Omega$.

\subsection{EEG Data Analysis}

Here for implementation and pre-processing the correction Technique of eye movement Brain Vision Analyzer a software was used offline for data analysis. The Math works (MATLAB) and EEGLAB were used for ICA and further processing. Raw data were band-pass filtered at $0.5-30 \mathrm{~Hz}$ after importing the data into the Visiona analyser software. For this purpose a phase shift free Butterworth filter (12 dB/Octave) is used. For detection of eye blinks threshold algorithm was conducted. At maximum blink excursion $800-1000 \mathrm{~ms}$ the data were segmented time-locked. After that a baseline correction was made at $-800:-500 \mathrm{~ms}$. Lastly, from occasional non blink-related artifacts the continuous raw data were cleaned. This was done by visual inspection

Table 1: Phi/Theta Coordinates M1 (System Full 112\%)

\begin{tabular}{|c|c|c|c|}
\hline & Theta, Phi & & Theta, Phi \\
\hline Fp1 & $-92,-72$ & F5 & $-75,-41$ \\
\hline Fp2 & 92,72 & F6 & 75,41 \\
\hline F3 & $-60,-51$ & FC5 & $-72,-21$ \\
\hline F4 & 60,51 & FC6 & 72,21 \\
\hline C3 & $-46,0$ & C5 & $-69,0$ \\
\hline C4 & 46,0 & C6 & 69,0 \\
\hline P3 & $-60,51$ & CP5 & $-72,21$ \\
\hline P4 & $60,-51$ & CP6 & $72,-21$ \\
\hline 01 & $-92,72$ & P5 & $-75,41$ \\
\hline 02 & $92,-72$ & P6 & $75,-41$ \\
\hline F7 & $-92,-36$ & AF7 & $-92,-54$ \\
\hline F8 & 92,36 & AF8 & 92,54 \\
\hline$\pi 7$ & $-92,0$ & $\mathrm{FT} 7$ & $-92,-18$ \\
\hline T8 & 92,0 & FT8 & 92,18 \\
\hline P7 & $-92,36$ & TP7 & $-92,18$ \\
\hline P8 & $92,-36$ & TP8 & $92,-18$ \\
\hline Fz & 46,90 & PO7 & $-92,54$ \\
\hline Cz & 0,0 & PO8 & $92,-54$ \\
\hline Pz & $46,-90$ & F9 & $-115,-36$ \\
\hline F1 & $-50,-68$ & F10 & 115,36 \\
\hline F2 & 50,68 & FT9 & $-115,-18$ \\
\hline FC1 & $-32,-45$ & FT10 & 115,18 \\
\hline $\mathrm{FC2}$ & 32,45 & TP9 & $-115,18$ \\
\hline c1 & $-23,0$ & TP10 & $115,-18$ \\
\hline $\mathrm{C} 2$ & 23,0 & P9 & $-115,36$ \\
\hline CP1 & $-32,45$ & P10 & $115,-36$ \\
\hline CP2 & $32,-45$ & PO9 & $-115,54$ \\
\hline P1 & $-50,68$ & P010 & $115,-54$ \\
\hline P2 & $50,-68$ & 09 & $-115,72$ \\
\hline AF3 & $-74,-65$ & 010 & $115,-72$ \\
\hline AF4 & 74,65 & Fpz & 92,90 \\
\hline FC3 & $-53,-33$ & AFZ & 69,90 \\
\hline FC4 & 53,33 & $\mathrm{FCz}$ & 23,90 \\
\hline CP3 & $-52,33$ & CPz & $23,-90$ \\
\hline CP4 & $52,-33$ & POZ & $69,-90$ \\
\hline PO3 & $-74,65$ & $\mathrm{Oz}$ & $92,-90$ \\
\hline PO4 & $74,-65$ & Iz & $115,-90$ \\
\hline
\end{tabular}

The residual activity after the blink maximum is another constraint. The components responsible for blink activity subtracted then the blink related activity remaining in the EEG signal is called artefactual. So the ocular artifact correction in ICA may not be focused only on the components responsible for horizontal eye movements or eye blinks. The technique ICA uses its power in decomposing multivariate data. It is a suitable tool to also remove the positivity mentioned. It is an important issue and need to be discussed further. When the decomposition is not perfect then during ICA conduction activity, the correlated temporal component with the blink is removed and we are violating the basic ICA assumptions due to data quality. The present results shows a pure blink signal is decomposed by ICA. This signal is residual independent. Because in the corrected simulated data and occurrence of residual does not take place. 


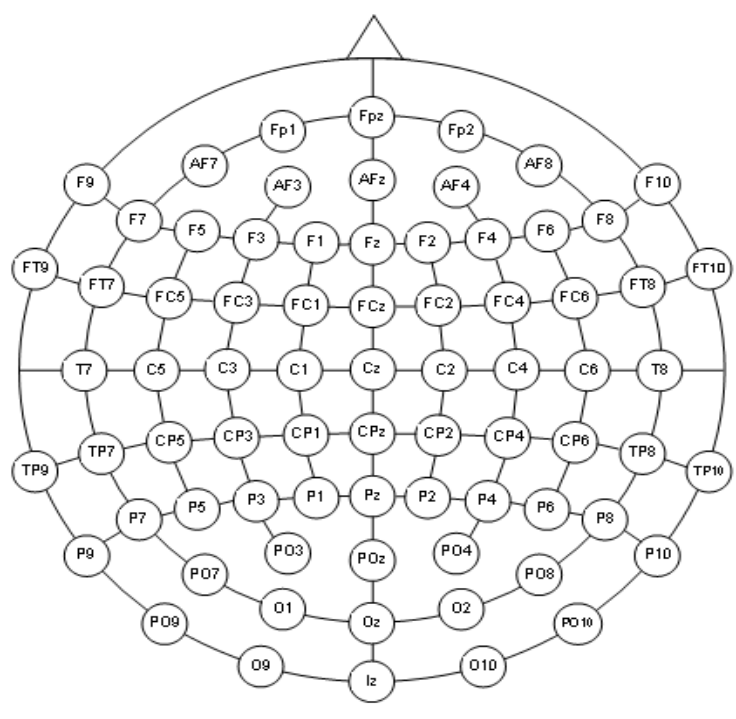

Figure 1.Theta/Phi-Coordinates M10 (Equidistant 61-ChannelArrangement

The ocular artefacts required further to analyze the corresponding components. It also need to make a detailed investigations and the dynamics of all different kinds of ocular activities. Different eye blinks at different situations for example refectory spontaneous and voluntary eye blinks carry different factors and different neural sources.[17]. These different situations may include intention, physical irritation, activation, cognitive load, or many other pathological reasons. These different factors or situations can be disentangled using ICA a powerful tool. It is a diversified tool and cannot be considered as a simple correction technique. Because the components responsible for natural artifacts and for components are independent from each other. If we just focus on the functional significance of derived components then the correction of the EEG signal will be obsolete. Finally, we conclude that ICA can remove eye blink artefects significantly. But there need a several possible algorithms for ICA technique to be operated upon for conduction. A systematic testing is required of these algorithms. We have also seen that there are many other powerful correction tools besides EMCP. There also exists component and regression based techniques. To evaluate a gain of new ICA correction procedures there require a systematic re-evaluation.

\section{Proposed Systems}

The EEG data obtained from the scalp of the patient is actually mixture of different levels of electrical potentials. Many temporally independent cerebral and artifactual bioelectrical processes generate these different electrical potentials. The sum of electrical potentials obtained from different sources is linear to all electrodes. Considering this we can write down the ICA model as follow

$E E G(t)=M \cdot S(t) \ldots \ldots .(1) "$

The cerebral bioelectrical processes which originate in the brain can be represented as

$$
c_{i, j}^{v}=\frac{n_{p}}{n_{i, j}} \ldots \ldots \text { (2) }
$$

The connectivity between the ROI's is the mean of the connectively $c_{i, j}^{R}=\frac{1}{n_{i} * n j} \sum_{k=1}^{n_{i}} \sum_{i=1}^{n_{j}} c_{k, l}$

The vertex degree ki of node $\mathrm{i}$ of the weighted network represent sum of all weights of edges being connected [20].

The vertex degree ki of node $i$ of the weighted network represent sum of all weights of edges being connected [20].

$$
k_{i}=\sum_{j} C_{i, j}^{R}
$$

To avoid the disconnection problem and compute the local cluster coefficient $\mathrm{Ci}$, average cluster coefficient $\mathrm{Cp}$,

$L_{p}=\frac{N *(N-1) / 2}{\sum_{i \neq j} 1 / d_{i, j}} \ldots$

\section{Results}

The results show that there an analogous impact on the mutual information of vertical EEG and EOG analogous impact with extended EMCP w/s. The Residual activity and infomax channels as well as the residual activity that remains maximum. For optimum the EMCP dependence is on the variance of blink activity. While ICA shows its independent data structure in such situations. Its important feature is that removal of blink artefacts is not the only characteristic. It analyze the EEG data and remove artefacts generally. Moreover, the ICA performance independent of the model which is considered the propagation or generation of EEG eye blink signal. The most important result is the late blink observance even after the correction technique applied.

This may not be taken as a a general conclusion against
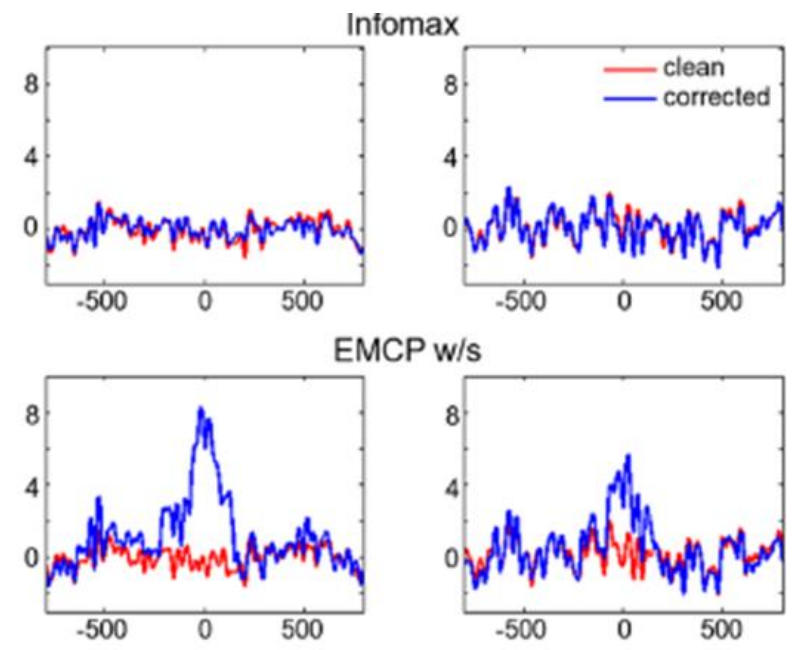

EMCPs

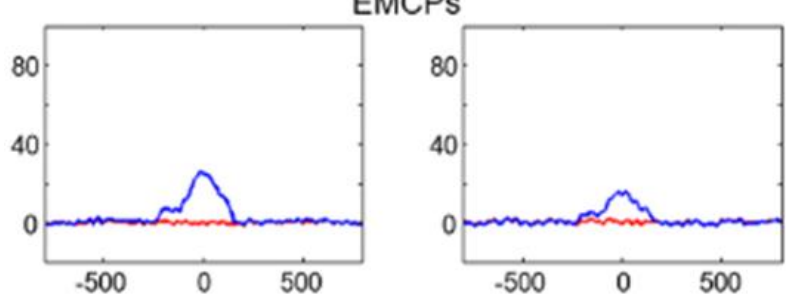

Figure 2. Propagation Model with randomly varying Blink Amplitude discussed regression based approaches.

The study is Fig.2 limited to eye blinks. With respect to horizontal and vertical eye movements more investigations are required. Several regression methods analysed earlier gives good 

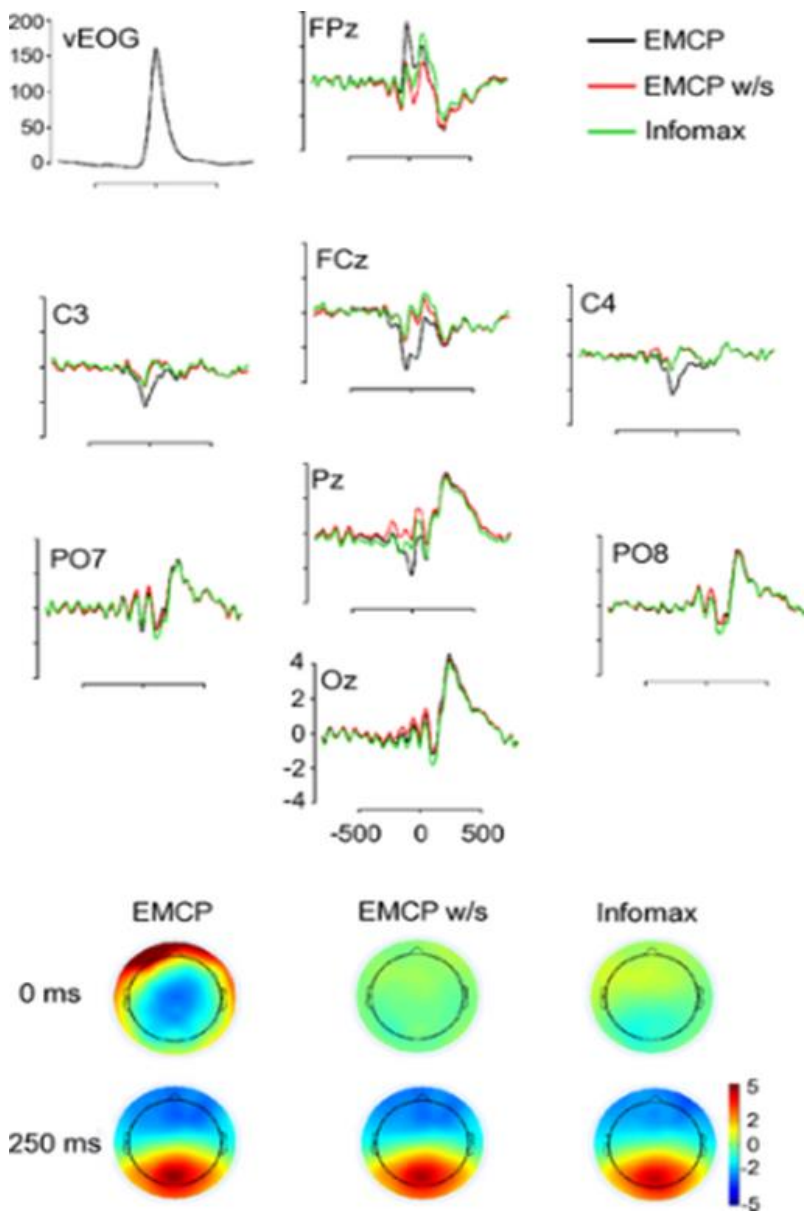

Figure 3. The Blink time averages of corrected EEG data
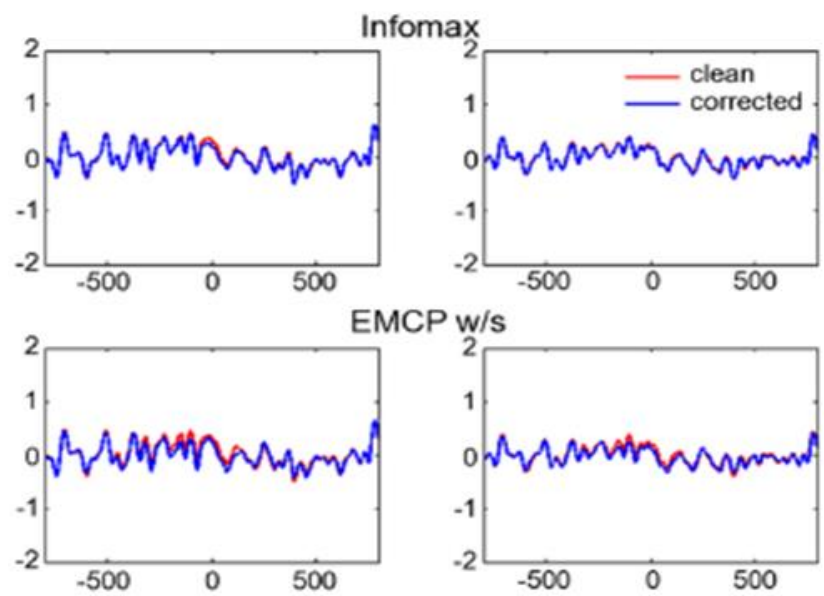

EMCP W/s

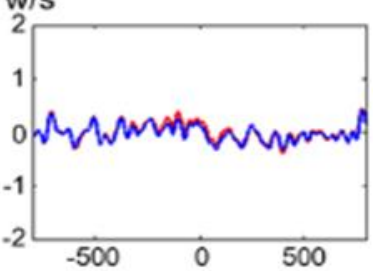

\section{EMCPs}
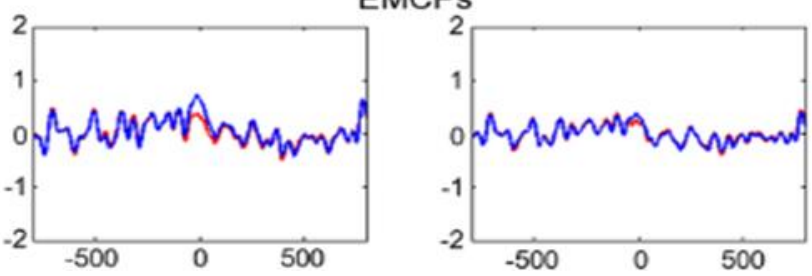

Figure 4. ICA Component Model Sgowing uncontaminated data

Figure 3 and 4 Results [13] shows the procedural results. Rather evaluating the impact of the EOG on the EEG after correction with two commonly used Techniques

For optimum the EMCP dependence is on the variance of blink activity. While ICA shows its independent data structure in such situations. Its important feature is that removal of blink artefacts is not the only characteristic. It analyse the EEG data and remove artefacts generally. Moreover, the ICA performance independent of the model which is considered the propagation or generation of EEG eye blink signal.

From results it is clear that there are different Differential impacts of different testing techniques on the real and the simulated data. Fcomponent Model for eye blinks the results are almost same as they are obtained from the real dataThe data corrected by EMCPs gives largest residual activity at maximum blink. But the ICAcorrected data and EMCP w/s data perfectly resemble the clean time course uncontaminated data. Figure 4 shows the locked averages at blink-time for the clean and uncontaminated data.

\section{Acknowledgements}

This study is is a research work in Biomedical Engineering using Image processing and signal processing. I hereby thanks Dr. Aamer Sleem chaudhury Professor at University of Lahore Islamabad Campus and Seema Tahir Lecturer at Department of Electrical Engineering Air University for their kind cooperation in research.

\section{References}

[1] Shumei Zhang, Paul McCullagh, Chris Nugent, Huiru Zheng and Matthias Baumgarten, "Optimal model selection for posture recognition in home-based healthcare", International Journal of Machine Learning and Cybernetics, Vol. 2, No. 1, pp. 1-14, 2011.

[2] Yi Tang, Pingkun Yan, Yuan Yuan and Xuelong Li, "Singleimage super-resolution via local learning", International Journal of Machine Learning and Cybernetics, Vol. 2, No. 1, pp. 15-23, 2011.

[3] Fadi N Karameh, Munther A. Dahleh, "Automated classification of EEG/ECG signals in tumor diagnostic", Proceedings of American control conference, Chicago, Illinois, June 2012.

[4] R. Verleger, T, Gasser, \& J. Mocks, "Correlation of EOG artifacts in eventrelated potentials of EEG: Aspects of reliability and validity" psychophysiology, Vol. 9, pp 472480, 2011.

[5] M. Murugesan, Mrs. R. Sukanesh “ Towards Detection of Brain Tumor in Electroencephalogram Signals using Support Vector Machines", International Journal of Computer Theory and Engineering, Vol. 1 No.5, December 2011.

[6] Shane M. Haas, Mark G. Frei, Ivan Osorio, Bozenna PasikDuncan, \& Jeff Radel, "EEG ocular artifact removal through ARMAX model system identification using extended least squares", Communication in Information and Systems , 3,(1), pp 19-40, 2003.

[7] M. Habl, Ch. Bauer, Ch. Ziegaus, E, W. Lang, F. Schulmeyer, "Can ICA help identify brain tumor related EEG signals?" International Workshop on Independent Component Analysis and Blind signal Separation, Helsinki, Finland, 19-22 June 2012.

[8] Alexander V. KRAMARENKO, Uner Tan, "Effects of High Frequency Electromagnetic Fields: A Brain Mapping Study", International Journal of Neuroscience, Vol. 113, pp. 1007-1019, 3003.

[9] Anthony J. Bell \& Terrance J. Sejnowski, “AN informationMaximization approach to Blind separation and Blind deconvolution ", Neural Computation, Vol 7 pp,1004-1034, 2011.

[10] Patange V.V \& Deshmukh B.T "O.C.R.-A Method of 
Computes and Works on the Vision of Scanned Image" International Journal of Electronics, Communication \& Instrumentation Engineering Research and Development, Vol. 2- 3, pp. 38-44, Sep. 30, 2012

[11] Chumman Lal, Chandarkar et al, "Survey of Image Contrast Enhancement Methods",International Journal of Electronics, Communication \& Instrumentation Engineering Research and Development, Vol. 2-3, pp. 56-63, Sep. 30, 2012.

[12] Usha Rani at al, "Image Developing Techniques -A Comparative Study", International Journal of Electronics, Communication \& Instrumentation Engineering Research and Development, Vol. 2-3,pp. 64-74, Sep. 30, 2012.

[13] B.Srevanthi \& C.H. Madhuri Devi, "Compressed Sensing Image Recovery Using Adaptive Nonlinear Filtering", International Journal of Electronics, Communication \& Instrumentation Engineering Research and Development, Vol. 2-3, pp. 75-80, Sep.30, 2012.

[14] AbhinavDeshpsnda \& S.K.Tadse, "Design Approach For Content-Based Image Recovery using Gabor-Zemike Features", International Journal of Electronics, Communication \& Instrumentation Engineering Research and Development, Vol.2-3 pp. 81-87, Sep. 30, 2012.

[15] Ms. P. Swati Sowjanya \& Ravi Mishra, "Video Shot Boundary Detection", International Journal of Electronics, Communication \& Instrumentation Engineering Research and Development, Vol.1-2, pp.72-83, Dec. 2011

[16] N. R. Pal and S. K. Pal , "A review on image segmentation techniques," Pattern Recognition, vol. 26, no. 9, pp. 12771294, 1993[2]

[17] L. P. Clarke, R. P. Velthuizen, M. A. Camacho, J. J. Heine, M.aidyanathan, L. O. Hall, R. W. Thatcher, and M. L. Silbiger, "MRI segmentation: methods and applications, Magn Reson Imaging , vol.13, no. 3, pp. 343$68,1995$.

[18] [Zhu, Xiaoping Li and Weiming Shen, "Effective genetic algorithm for resource-constrained project scheduling with limited preemptions", International Journal of Machine Learning and Cybernetics, March 2011. DOI: 10.1007/s1 3042-011-0014-3.

[19] Shumei Zhang, Paul McCullagh, Chris Nugent, Huiru Zheng and Matthias Baumgarten, "Optimal model selection for posture recognition in home-based healthcare", International Journal of Machine Learning and Cybernetics, Vol. 2, No. 1, pp. 1-14, 2011.

[20] Yi Tang, Pingkun Yan, Yuan Yuan and Xuelong Li, "Singleimage super-resolution via local learning", International Journal of Machine Learning and Cybernetics, Vol. 2, No. 1, pp. 15-23, 2011

[21] Fadi N Karameh, Munther A. Dahleh, "Automated classification of EEG/ECG signals in tumor diagnostic" , Proceedings of American control conference, Chicago, Illinois, June 2012.

[22] R. Verleger, T, Gasser, \& J. Mocks, "Correlation of EOG artifacts in eventrelated potentials of EEG: Aspects of reliability and validity", psychophysiology, Vol. 9, pp 472480, 2011

[23] M. Murugesan, Mrs. R. Sukanesh “ Towards Detection of Brain Tumor in Electroencephalogram Signals using Support Vector Machines", International Journal of Computer Theory and Engineering, Vol. 1 No.5, December 2011.

[24] Shane M. Haas, Mark G. Frei, Ivan Osorio, Bozenna PasikDuncan, \& Jeff Radel, "EEG ocular artifact removal through ARMAX model system identification using extended least squares", Communication in Information and Systems , 3,(1), pp 19-40, 2003.

[25] Akram Rashid, Zahooruddin and Dr. I.M. Qureshi "Electrocardiogram Signal Processing for baseline Noise Removal using Blind Source Separation Techniques: A Comparative Analysis" IEEE Trans on Machine Learning and Cybernetics Guilin, Guangxi, China July 2011. Volume 4, pp1756

[26] Akram Rashid, Zahooruddin, Ijaz Mansoor Qureshi, Aamer Saleem "Electrocardiogram Signal Processing for Baseline Noise Removal USING Blind source separation techniques" International Conference on Machine Learning and Cybernetics, Vol. 4, pp 1756-1761, 2011

[27] Akram Rashid "Multiuser Detection in DS-CDMA using Evolutionary Techniques"International Conference on Computer, Electrical, System Science and Engineering, France Paris July 28-30. 2010. 